\title{
Azimuthal-angle Observables in Inclusive Three-jet Production
}

\section{G. Chachamis*}

Instituto de Física Teórica UAM/CSIC, Nicolás Cabrera 15

\& Universidad Autónoma de Madrid, E-28049 Madrid, Spain. E-mail:

chachamis@gmail. com

\section{F. Caporale}

Instituto de Física Teórica UAM/CSIC, Nicolás Cabrera 15

\& Universidad Autónoma de Madrid, E-28049 Madrid, Spain. E-mail:

francesco.caporale@uam.es

\section{F. G. Celiberto}

Dipartimento di Fisica, Università della Calabria \&

Istituto Nazionale di Fisica Nucleare, Gruppo Collegato di Cosenza,

I-87036 Arcavacata di Rende, Cosenza, Italy. E-mail:

francescogiovanni.celiberto@fis.unical.it

D. Gordo Gómez

Instituto de Física Teórica UAM/CSIC, Nicolás Cabrera 15

\& Universidad Autónoma de Madrid, E-28049 Madrid, Spain. E-mail:

david.gordo@csic.es

\section{A. Sabio Vera}

Instituto de Física Teórica UAM/CSIC, Nicolás Cabrera 15

\& Universidad Autónoma de Madrid, E-28049 Madrid, Spain. E-mail:

a.sabio.vera@gmail.com

\begin{abstract}
We discuss the impact of corrections beyond the leading-logarithmic accuracy on some recently proposed LHC observables that are based on azimuthal-angle ratios in a kinematical setup that is an extension to the usual one for Mueller-Navelet jets, after requiring an extra tagged jet in central regions of rapidity. The corrections tend to be mild which suggests that these observables are an excellent way to probe the onset of BFKL effects at hadronic colliders.
\end{abstract}

25th International Workshop on Deep Inelastic Scattering and Related Topics

3-7 April 2017

University of Birmingham, Birmingham, UK

\footnotetext{
* Speaker.

${ }^{\dagger}$ La Caixa-Severo Ochoa Scholar.
} 


\section{Introduction}

In high energy scattering in Quantum Chromodynamics (QCD) the Balitsky-Fadin-KuraevLipatov (BFKL) framework in the leading logarithmic (LLA) [1-6] and next-to-leading logarithmic (NLLA) approximation $[7,8]$ are used for the resummation of large logarithms in the center-of-mass energy squared $s$

An interesting case is Mueller-Navelet jets [9], configurations with two final state jets ${ }^{1}$ with transverse momenta of similar sizes, $k_{A, B}$, and a rapidity distance $Y=\ln \left(x_{1} x_{2} s /\left(k_{A} k_{B}\right)\right)$ large. A number of studies [13-28] focused on the azimuthal angle $(\theta)$ behaviour of the two jets regards that the presence of decisive minijet activity in the rapidity space between these two outermost jets can be accounted for by a BFKL gluon Green function connecting the two jets. Moreover, it was shown $[29,30]$, that ratios of projections on azimuthal angle observables

$$
\mathscr{R}_{n}^{m}=\langle\cos (m \theta)\rangle /\langle\cos (n \theta)\rangle,
$$

(where $m, n$ are integers) are much more favourable quantities in the search for a clear signal of BFKL effects. The comparison of different NLLA calculations for these ratios $\mathscr{R}_{n}^{m}$ [31-35] against LHC experimental data has been promising.

Along these lines, new observables were recently proposed for processes with three-jet $[36,37]$ and four-jet final states $[38,39]$ with the outermost jets having a large rapidity distance and any other tagged jet is to be found in more central regions of the detector. Here we will restrict the discussion to inclusive three-jet production assuming that the jets are connected in the $t$-channel via gluon Green's functions. The main idea presented in Refs. [36,37] was to produce theoretical estimates for the ratios

$$
R_{P Q}^{M N}=\frac{\left\langle\cos \left(M \theta_{1}\right) \cos \left(N \theta_{2}\right)\right\rangle}{\left\langle\cos \left(P \theta_{1}\right) \cos \left(Q \theta_{2}\right)\right\rangle}=\frac{C_{M N}}{C_{P Q}}
$$

where $\theta_{1}$ is the azimuthal angle difference between the first and the second (central) jet, while, $\theta_{2}$ is the azimuthal angle difference between the second and the third jet. In this work, we show theoretical predictions for $R_{33}^{22}$ at NLLA, originally presented in [40]. We are interested in seeing whether we have large corrections once we consider the NLLA gluon Green's functions instead of the leading-logarithmic accuracy ones. Large corrections of that origin is not a surprising outcome for many BFKL-based calculations and they could potentially have a strong impact on the ratios $R_{P Q}^{M N}$, hence they need to be addressed.

We assume for the outermost jet transverse momenta that $k_{A}^{\min }=35 \mathrm{GeV}, k_{B}^{\min }=50 \mathrm{GeV}$, $k_{A}^{\max }=k_{B}^{\max }=60 \mathrm{GeV}$, whereas the transverse momentum of the central jet, $k_{J}$, can live in three wide bins, that is, $20 \mathrm{GeV}<k_{J}<35 \mathrm{GeV}$ (bin-1), $35 \mathrm{GeV}<k_{J}<60 \mathrm{GeV}$ (bin-2) and $60 \mathrm{GeV}<$ $k_{J}<120 \mathrm{GeV}$ (bin-3). To quantify the difference between LLA and NLLA, we define

$$
\delta x(\%)=\left(\operatorname{res}^{(\mathrm{LLA})}-\frac{\mathrm{res}^{(\mathrm{BLM}-1)}+\mathrm{res}^{(\mathrm{BLM}-2)}}{2}\right) \frac{1}{\mathrm{res}^{(\mathrm{LLA})}},
$$

\footnotetext{
${ }^{1}$ Another interesting idea, suggested in [10] and investigated in [11,12], is the study of the production of two charged light hadrons, $\pi^{ \pm}, K^{ \pm}, p, \bar{p}$, with large transverse momenta and well separated in rapidity.
} 
where res ${ }^{(\mathrm{LLA})}$ is the LLA result while res ${ }^{(\mathrm{BLM}-1)}$ and res ${ }^{(\mathrm{BLM}-2)}$ are NLLA results in the BLM scheme [41] that differ in the renormalisation scale to give us a measure of the theoretical uncertainty.

\section{Results}

First we present results for $R_{33}^{22}$ as a function of the rapidity difference after integrating over a central jet rapidity bin, that is, after allowing for the central jet rapidity to take values in the range $-0.5<y_{J}<0.5$, see Fig. 1 . We use dashed lines to represent LLA results and a band for the NLLA results. The band is bounded by the res ${ }^{(\mathrm{BLM}-1)}$ and res ${ }^{(\mathrm{BLM}-2)}$ results in thin continuous lines. The red curve and band are representing the LLA and NLLA results respectively for the central jet belonging to bin- 1 the green curve and band for the central jet belonging to bin- 2 and the blue curve and band are representing the LLA and NLLA results respectively for the central jet belonging to bin-3. We see that the NLLA corrections are mild. Moreover, we note that the overall picture does not change considerably when we go from LLA to NLLA.
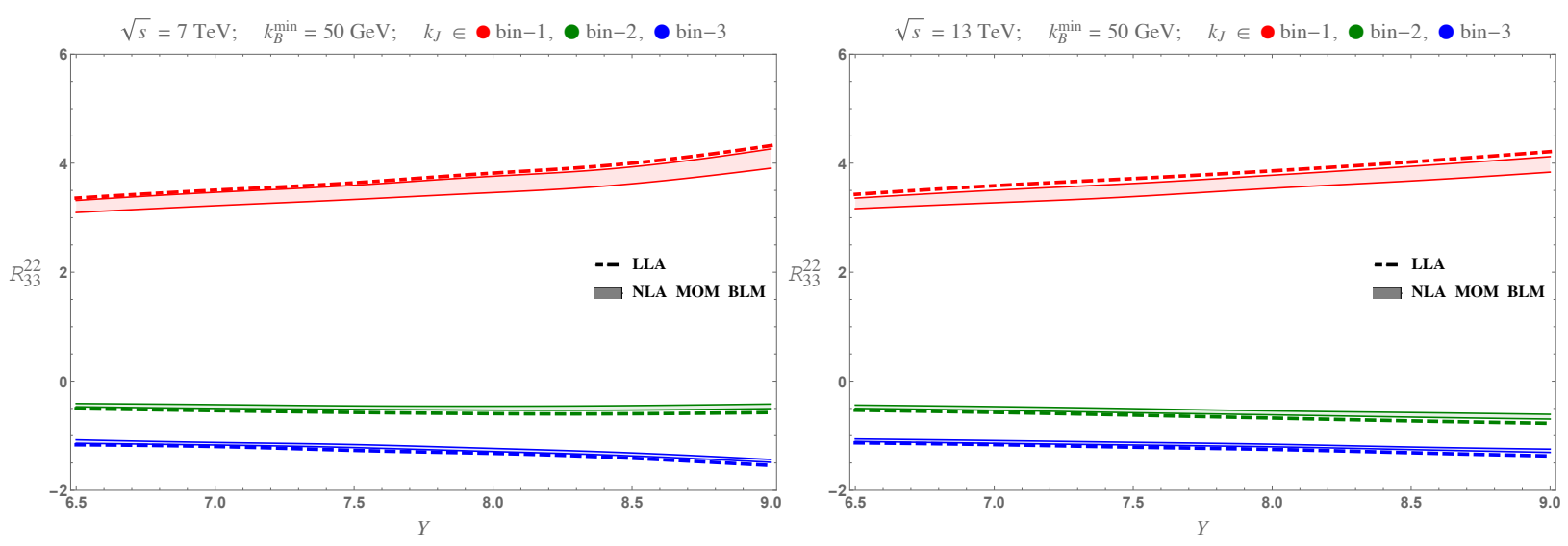

Figure 1: $Y$-dependence of the LLA (dashed lines) and NLLA (bands) results for $R_{33}^{22}$ with $y_{J}$ integrated over a central rapidity bin at $\sqrt{s}=7 \mathrm{TeV}$ (left) and $\sqrt{s}=13 \mathrm{TeV}$ (right).

Next, in Fig. 2, we allow for $Y_{A}$ (rapidity of the forward jet) and $Y_{B}$ (rapidity of the backward jet) to take values such that $\left(Y_{A}^{\min }=3\right)<Y_{A}<\left(Y_{A}^{\max }=4.7\right)$ and $\left(Y_{B}^{\min }=-4.7\right)<Y_{B}<\left(Y_{B}^{\max }=\right.$ $-3)$. Furthermore, the rapidity of the central jet can take values in five distinct rapidity bins of unit width, that is, $y_{i}-0.5<y_{J}<y_{i}+0.5$, with $y_{i}=\{-1,-0.5,0,0.5,1\}$. Obviously, the ratios now are functions of $y_{i}$, namely,

$$
R_{P Q}^{M N}\left(y_{i}\right)=\frac{C_{M N}^{\mathrm{integ}}\left(y_{i}\right)}{C_{P Q}^{\mathrm{integ}}\left(y_{i}\right)} .
$$

We see that the $y_{i}$-dependence of the three ratios is very weak. Moreover, the similarity between the $\sqrt{s}=7 \mathrm{TeV}$ and $\sqrt{s}=13 \mathrm{TeV}$ plots is more striking that in the Fig. 1 and the NLLA corrections seem even milder. 

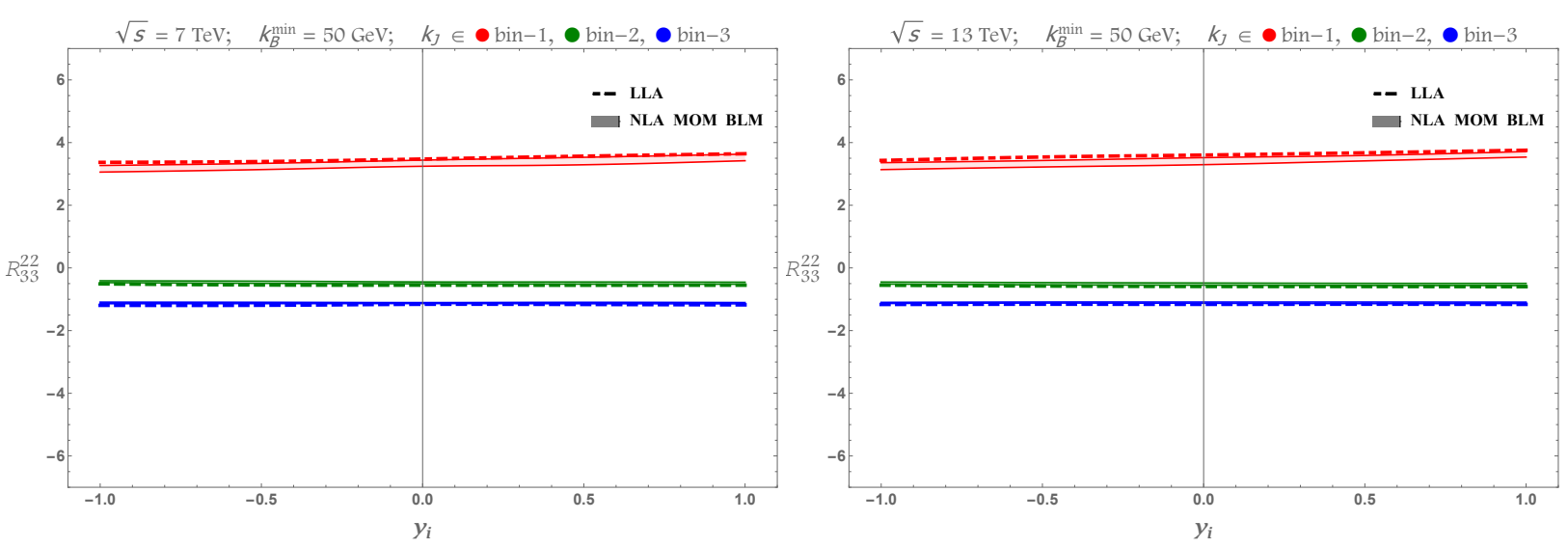

Figure 2: $y_{i}$-dependence of the LLA (dashed lines) and NLLA (bands) results for $R_{33}^{22}$ at $\sqrt{s}=7 \mathrm{TeV}$ (left) and $\sqrt{s}=13 \mathrm{TeV}$ (right).

\section{Summary \& Outlook}

We have shown some results from a first beyond the leading logarithmic accuracy work on generalised azimuthal-angle observables in inclusive three-jet production at the LHC within the BFKL formalism. In addition, for a proper study of the total corrections beyond the LLA, the NLO jet vertices need to be included and the NLLA gluon Green functions.

Our most important conclusion in this work is that the corrections that come into play after considering NLLA gluon Green's functions are generally mild and the generalised ratio observables exhibit perturbative stability. Moreover, we note that the differences in the plots for $7 \mathrm{TeV}$ to $13 \mathrm{TeV}$ are small which suggests that these observables capture the crucial features of the BFKL dynamics with regard to the azimuthal behavior of the hard jets in hadronic inclusive three-jet production. We plan to compare our results here against theoretical estimates for these observables from fixed order calculations, the full BFKL Monte Carlo BFKLex [42-49] as well as from general-purpose Monte Carlos tools.

\section{Acknowledgements}

This work was supported by the Spanish Research Agency (Agencia Estatal de Investigación) through the grant IFT Centro de Excelencia Severo Ochoa SEV-2016-0597. GC and ASV acknowledge support from the MICINN, Spain, under contract FPA2016-78022-P. DGG is supported with a fellowship of the international programme "La Caixa-Severo Ochoa".

\section{References}

[1] L. N. Lipatov, Sov. Phys. JETP 63 (1986) 904 [Zh. Eksp. Teor. Fiz. 90 (1986) 1536].

[2] I. I. Balitsky and L. N. Lipatov, Sov. J. Nucl. Phys. 28 (1978) 822 [Yad. Fiz. 28 (1978) 1597].

[3] E. A. Kuraev, L. N. Lipatov and V. S. Fadin, Sov. Phys. JETP 45 (1977) 199 [Zh. Eksp. Teor. Fiz. 72 (1977) 377].

[4] E. A. Kuraev, L. N. Lipatov and V. S. Fadin, Sov. Phys. JETP 44 (1976) 443 [Zh. Eksp. Teor. Fiz. 71 (1976) 840] [Erratum-ibid. 45 (1977) 199]. 
[5] L. N. Lipatov, Sov. J. Nucl. Phys. 23 (1976) 338 [Yad. Fiz. 23 (1976) 642].

[6] V. S. Fadin, E. A. Kuraev and L. N. Lipatov, Phys. Lett. B 60 (1975) 50.

[7] V. S. Fadin and L. N. Lipatov, Phys. Lett. B 429 (1998) 127 [hep-ph/9802290].

[8] M. Ciafaloni and G. Camici, Phys. Lett. B 430 (1998) 349 [hep-ph/9803389].

[9] A. H. Mueller and H. Navelet, Nucl. Phys. B 282 (1987) 727.

[10] D. Y. Ivanov and A. Papa, JHEP 1207, 045 (2012) [arXiv:1205.6068 [hep-ph]].

[11] F. G. Celiberto, D. Y. Ivanov, B. Murdaca and A. Papa, Phys. Rev. D 94, no. 3, 034013 (2016) doi:10.1103/PhysRevD.94.034013 [arXiv:1604.08013 [hep-ph]].

[12] F. G. Celiberto, D. Y. Ivanov, B. Murdaca and A. Papa, Eur. Phys. J. C 77, no. 6, 382 (2017) doi:10.1140/epjc/s10052-017-4949-8 [arXiv:1701.05077 [hep-ph]].

[13] V. Del Duca and C. R. Schmidt, Phys. Rev. D 49 (1994) 4510 [hep-ph/9311290].

[14] W. J. Stirling, Nucl. Phys. B 423 (1994) 56 [hep-ph/9401266].

[15] L. H. Orr and W. J. Stirling, Phys. Rev. D 56 (1997) 5875 [hep-ph/9706529].

[16] J. Kwiecinski, A. D. Martin, L. Motyka and J. Outhwaite, Phys. Lett. B 514 (2001) 355 [hep-ph/0105039].

[17] J. R. Andersen et al. [Small x Collaboration], Eur. Phys. J. C 48, 53 (2006) doi:10.1140/epjc/s2006-02615-6 [hep-ph/0604189].

[18] A. De Roeck et al., Eur. Phys. J. C 66, 525 (2010) doi:10.1140/epjc/s10052-010-1244-3 [arXiv:0909.3240 [hep-ph]].

[19] M. Angioni, G. Chachamis, J. D. Madrigal and A. Sabio Vera, Phys. Rev. Lett. 107, 191601 (2011) [arXiv:1106.6172 [hep-th]].

[20] F. Caporale, B. Murdaca, A. Sabio Vera and C. Salas, Nucl. Phys. B 875 (2013) 134 [arXiv:1305.4620 [hep-ph]].

[21] F. Caporale, D. Y. Ivanov, B. Murdaca and A. Papa, Nucl. Phys. B 877 (2013) 73 [arXiv:1211.7225 [hep-ph]].

[22] C. Marquet and C. Royon, Phys. Rev. D 79, 034028 (2009) [arXiv:0704.3409 [hep-ph]].

[23] D. Colferai, F. Schwennsen, L. Szymanowski and S. Wallon, JHEP 1012, 026 (2010) [arXiv:1002.1365 [hep-ph]].

[24] B. Ducloue, L. Szymanowski and S. Wallon, JHEP 1305, 096 (2013) [arXiv:1302.7012 [hep-ph]].

[25] B. Ducloue, L. Szymanowski and S. Wallon, Phys. Lett. B 738, 311 (2014) [arXiv:1407.6593 [hep-ph]].

[26] A. H. Mueller, L. Szymanowski, S. Wallon, B. W. Xiao and F. Yuan, JHEP 1603, 096 (2016) [arXiv:1512.07127 [hep-ph]].

[27] G. Chachamis, arXiv:1512.04430 [hep-ph].

[28] K. Akiba et al. [LHC Forward Physics Working Group Collaboration], J. Phys. G 43, 110201 (2016) [arXiv:1611.05079 [hep-ph]].

[29] A. Sabio Vera, Nucl. Phys. B 746 (2006) 1 [hep-ph/0602250]. 
[30] A. Sabio Vera and F. Schwennsen, Nucl. Phys. B 776 (2007) 170 [hep-ph/0702158 [HEP-PH]].

[31] B. Ducloue, L. Szymanowski and S. Wallon, Phys. Rev. Lett. 112 (2014) 082003 [arXiv:1309.3229 [hep-ph]].

[32] F. Caporale, D. Y. Ivanov, B. Murdaca and A. Papa, Eur. Phys. J. C 74 (2014) 3084 [arXiv:1407.8431 [hep-ph]].

[33] F. Caporale, D. Y. Ivanov, B. Murdaca and A. Papa, Phys. Rev. D91 (2015) 11, 114009 [arXiv:1504.06471 [hep-ph]].

[34] F. G. Celiberto, D. Yu. Ivanov, B. Murdaca and A. Papa, Eur. Phys. J. C 75 (2015) 292 [arXiv: 1504.08233 [hep-ph]].

[35] F. G. Celiberto, D. Y. Ivanov, B. Murdaca and A. Papa, Eur. Phys. J. C 76, no. 4, 224 (2016) doi:10.1140/epjc/s10052-016-4053-5 [arXiv:1601.07847 [hep-ph]].

[36] F. Caporale, G. Chachamis, B. Murdaca and A. Sabio Vera, Phys. Rev. Lett. 116, no. 1, 012001 (2016) [arXiv:1508.07711 [hep-ph]].

[37] F. Caporale, F. G. Celiberto, G. Chachamis, D. Gordo Gomez and A. Sabio Vera, Nucl. Phys. B 910, 374 (2016) doi:10.1016/j.nuclphysb.2016.07.012 [arXiv:1603.07785 [hep-ph]].

[38] F. Caporale, F. G. Celiberto, G. Chachamis and A. S. Vera, Eur. Phys. J. C 76, no. 3, 165 (2016) [arXiv:1512.03364 [hep-ph]].

[39] F. Caporale, F. G. Celiberto, G. Chachamis, D. Gordo Gómez and A. Sabio Vera, Eur. Phys. J. C 77, no. 1, 5 (2017) doi:10.1140/epjc/s10052-016-4557-z [arXiv:1606.00574 [hep-ph]].

[40] F. Caporale, F. G. Celiberto, G. Chachamis, D. G. Gomez and A. Sabio Vera, Phys. Rev. D 95, no. 7, 074007 (2017) doi:10.1103/PhysRevD.95.074007 [arXiv:1612.05428 [hep-ph]].

[41] S.J. Brodsky, G.P. Lepage, P.B. Mackenzie, Phys. Rev. D 28, 228 (1983).

[42] G. Chachamis, M. Deak, A. Sabio Vera and P. Stephens, Nucl. Phys. B 849 (2011) 28 [arXiv:1102.1890 [hep-ph]].

[43] G. Chachamis and A. Sabio Vera, Phys. Lett. B 709 (2012) 301 [arXiv:1112.4162 [hep-th]].

[44] G. Chachamis and A. Sabio Vera, Phys. Lett. B 717 (2012) 458 [arXiv:1206.3140 [hep-th]].

[45] G. Chachamis, A. Sabio Vera and C. Salas, Phys. Rev. D 87 (2013) 1, 016007 [arXiv:1211.6332 [hep-ph]].

[46] F. Caporale, G. Chachamis, J. D. Madrigal, B. Murdaca and A. Sabio Vera, Phys. Lett. B 724 (2013) 127 [arXiv:1305.1474 [hep-th]].

[47] G. Chachamis and A. Sabio Vera, Phys. Rev. D 93, no. 7, 074004 (2016) [arXiv:1511.03548 [hep-ph]].

[48] G. Chachamis and A. Sabio Vera, JHEP 1602 (2016) 064 [arXiv:1512.03603 [hep-ph]].

[49] G. Chachamis and A. Sabio Vera, Phys. Rev. D 94, no. 3, 034019 (2016) [arXiv:1606.07349 [hep-ph]]. 